\title{
Novel Cell-based Biosensing with 2D-SPR imager
}

\author{
Hiroaki Shinohara \\ Graduate School of Science and Engineering, University of Toyama, \\ 3190 Gofuku, Toyama, 930-8555 Japan, \\ e-mail: hshinoha@eng.u-toyama.ac.jp
}

\begin{abstract}
:
In this paper, novel cell-based biosensing system with a high resolution of two dimensional surface plasmon resonance (2D-SPR) imager is introduced. Response of a model nerve precursor cell (PC12) which was adhered on an Au chip to agonist stimulation was observed by the reflection intensity change in the individual cell regions with the 2D-SPR imager at real-time without any probe reagents. It was further demonstrated that the cell response in 2D-SPR imaging to agonist stimulation might be based on intracellular recruitment of signal transduction proteins such as PKC to cell membrane. These results suggested future wide applications of label-free cell-based biosensing with 2D-SPR imager, such as drug screening, cell state evaluation and so on.
\end{abstract}

Key words: surface plasmon resonance (SPR), imaging, culture cells, cell response, PKC translocation and drug screening.

\section{Introduction}

2D-SPR imaging has been applied to simultaneous multi-sensing for target proteins or DNAs by using antigen-antibody reaction or DNA hybridization reaction. On the other hand, we have recently applied a high resolution of 2D-SPR imager to observe the responses of RBL-2H3 cells as a model mast cell to allergen stimulation and succeeded in allergen sensing with high sensitivity $[1,2$,$] . Furthermore, we$ have previously observed the response of PC12 cell as a model nerve precursor cell to high $\mathrm{K}^{+}$stimulation with the 2D-SPR imager [3].

In this paper, to introduce usefulness and development of cell-based 2D-SPR biosensing, PC12 cell responses to the acetylcholine receptor agonists were observed with a high resolution $2 \mathrm{D}-\mathrm{SPR}$ imager. The agonist sensing mechanism with this system, i.e. "What intracellular reaction by agonist was observed with the 2D-SPR imager?" is also discussed.

\section{Experimental Method:}

\subsection{D-SPR imaging system}

2D-SPR sensor (NTT-AT, Japan) with a collimator to parallelize incident light, a cooling CCD camera to obtain reflection image and four kinds of magnification lens $(1 x, 2 x, x 4,7 x)$ to take high resolution image was used for the 2D-SPR observation. Fig. 1 shows schematic illustration of the 2D-SPR observation system used for cell response imaging.

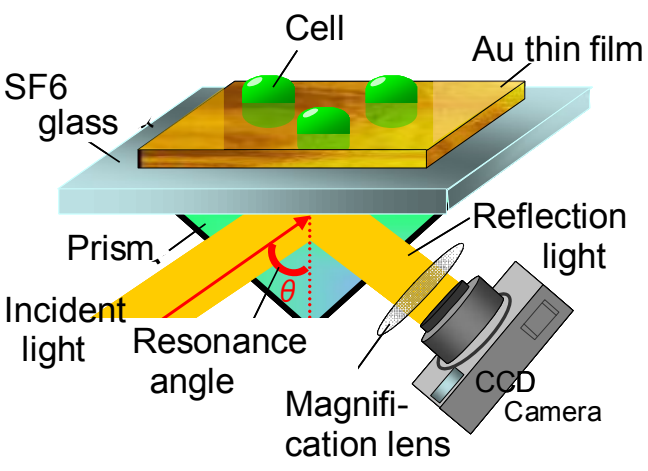

Fig. 1 Schematic illustration of the 2D-SPR imaging system for observation of cell response.

\subsection{Cell culture and $2 D-S P R$ observation}

PC12 cell was cultured on $50 \mathrm{~nm}$ gold-coated SF6 glass chip $(18 \times 18 \mathrm{~mm})$ with a silicon well (flexiPERM) over 1day in a humidified atmosphere containing $5 \% \mathrm{CO}_{2}$ at $37^{\circ} \mathrm{C}$. The sensor chip on which cells are adhered was set on the prism of the 2D-SPR imager with matching oil and reflection image of P-polarized parallel incident light was recorded. SPR curve that is dependence of reflection intensity of the region of interest on incident angle was first measured to find resonance angle of the region and to determine suitable measurement angle. Time course of cell response for stimulant in 2D-SPR imaging was monitored at the suitable measurement angle. Phorbol-12-myristate-13acetate (PMA) and staurosporin was used to consider the contribution of intracellular PKC translocation to the SPR response. 


\section{Results and Discussion}

\section{3-1. 2D-SPR observation of responses of $P C 12$ cells to muscarine stimulation}

Rat pheochromocytoma PC12 cell was used as a model nerve precursor cell which has muscarinic acetylcholine receptors on the cell membrane. 2D-SPR images of PC12 cell regions before and after stimulation by muscarine which is well known to induce PKC translocation in PC12 cell are shown in Fig.2. By muscarine injection, almost cell regions brightened, whereas a control region (Au surface) showed no change. Time course of the reflection intensity in the individual cell regions is shown in Fig.3. Reflection intensity of the almost cell regions showed slow but significant increase within $10 \mathrm{~min}$.

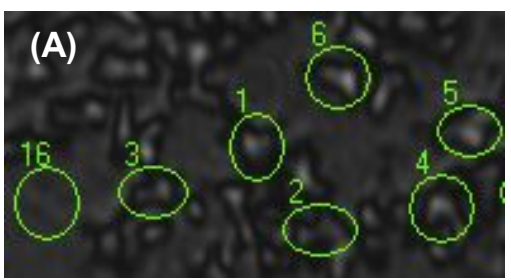

(A) Before muscarine stimulation.

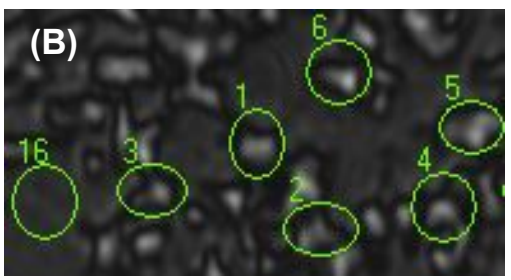

(B) $20 \mathrm{~min}$ after $100 \mu \mathrm{M}$ muscarine stimulation.

No.1 6 are cell regions and No.16 is a control $(A u)$ region.

Fig.2. 2D-SPR images of PC12 cell regions.

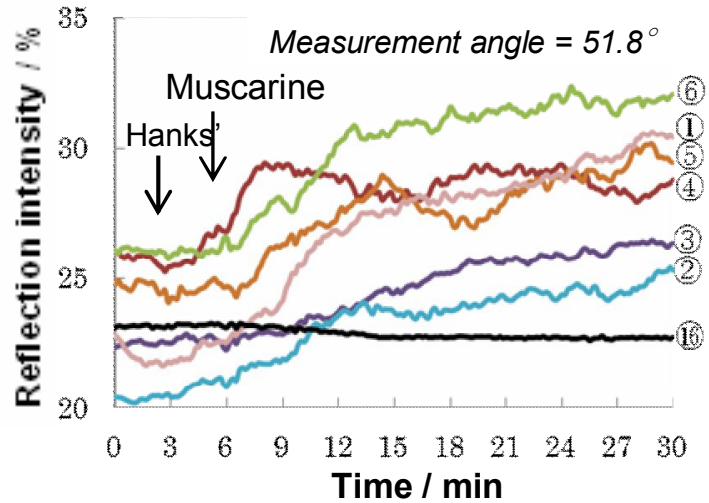

Fig.3. Time course of reflection intensity of individual cell regions (1 6) and a control region upon $100 \mu \mathrm{M}$ muscarine stimulation.

Next, the dependence of the reflection intensity increase on the muscarine concentration was investigated as shown in Fig.4. Muscarine detection was performed from 5 to $50 \mu \mathrm{M}$.

\section{3-2. What intracellular reaction by agonist was observed with the $2 D-S P R$ imager?}

Slow and concentration-dependent increase of reflection intensity at the PC12 cell regions was

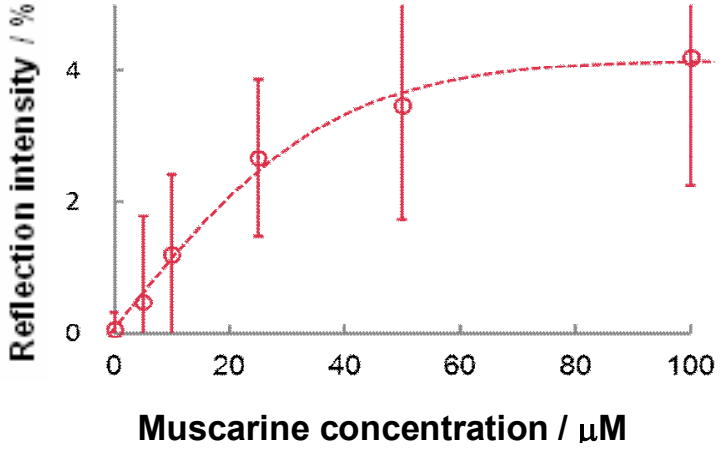

Fig.4. Muscarine sensing by the PC12cell response.

observed with the 2D-SPR imager by injection of PMA which activate PKC directly. Furthermore, the addition of 100nM staurosporine, a PKC inhibitor, prior to PMA injection suppressed significantly the reflection intensity increase as shown in Fig.5. These data strongly suggested that the SPR sensed the PKC translocation from cytosol to the adhered cell membrane as a major reaction.

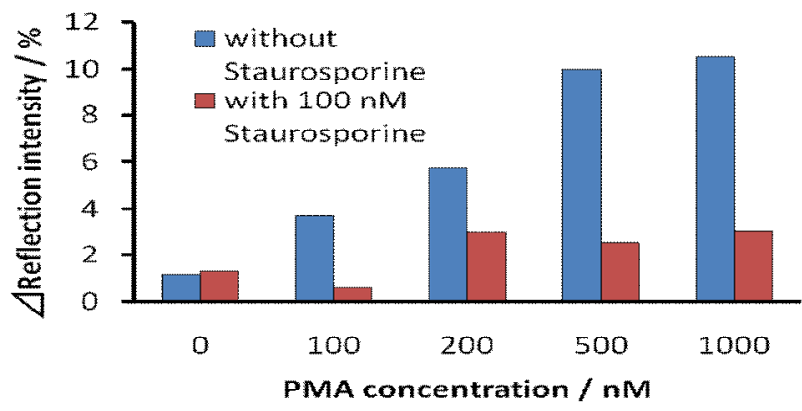

Fig.5. Reflection intensity change of PC12 cell regions by injection of various conc. of PMA and the inhibition by preceded 100nM staurosporine addition.

\section{Conclusion}

We would like to emphasize that 2D-SPR imaging is very useful to observe individual cell responses without any probe reagents and for easy, sensitive sensing for the cell stimulants which induce PKC translocation.

\section{Acknowledgements}

This study was supported by MEXT/JSPS KAKENHI Grant No.23360365.

\section{References}

[1] M. Horii, H. Shinohara, Y. Iribe, and M. Suzuki, The Proceedings of $\mu$ TAS 2007 Conference, pp.451-453, Oct. 7-11, 2007, Paris, France.

[2] M. Horii, H. Shinohara, Y. Irebe, M. Suzuki, Analyst, 136, 2706-2711(2011); DOI: 10. 1039/c0an00874e.

[3] H. Shinohara, Y. Sakai, and M. Suzuki, Technical Digest of the $13^{\text {th }}$ International Meeting on Chemical Sensors, p.82, July11-14, 2010, Perth, Australia. 